\title{
Synchronization of Discrete-Time Complex Networks with Randomly Occurring Coupling and Distributed Time-Varying Delay
}

\author{
Yang Tang ${ }^{1,2}$ and Jian-an Fang ${ }^{1}$ \\ ${ }^{1}$ College of Information Science and Technology, Donghua University, Shanghai 201620, China \\ ${ }^{2}$ Institute of Textiles and Clothing, Kowloon, Hong Kong Polytechnic University, Hong Kong \\ Correspondence should be addressed to Yang Tang, tangtany@gmail.com
}

Received 6 December 2009; Accepted 19 March 2010

Academic Editor: Ağacik Zafer

Copyright (C) 2010 Y. Tang and J.-a. Fang. This is an open access article distributed under the Creative Commons Attribution License, which permits unrestricted use, distribution, and reproduction in any medium, provided the original work is properly cited.

The synchronization problem of new discrete-time complex networks with randomly occurring coupling and distributed time-varying delay is investigated. Compared with the previous work, the distributed delay is assumed to be timevarying. Moreover, the effects of both variation range and probability distribution of distributed time-varying delay are taken into consideration. The randomly occurring coupling and distributed delay in complex networks are considered by introducing two Bernoulli stochastic variables. By using some novel analysis techniques, Kronecker product and Lyapunov functional candidate, some delay-distribution-dependent conditions are derived to ensure that the discrete-time complex network with randomly coupling and distributed time-varying delay is synchronized in mean square. A numerical simulation example is provided to verify the effectiveness and the applicability of the proposed approach.

\section{Introduction}

Synchronization is an important property of complex networks, which can be seen in natural, social, physical and biological fields, and has been found applications in everywhere of real world [1-17]. In engineering applications such as network-based control, time-series analysis, and image processing, it is often necessary to formulate a discrete-time analog of the continuous-time networks. Recently, synchronization of discrete-time complex networks has drawn much interests $[8,9,11-14]$.

In practical situations, timedelays in complex networks are necessary to be taken into account for modeling a realistic networks since the information transmission within complex networks is in general not instantaneous [18-28]. Synchronization problem of complex 
networks with timedelay has been investigated by many researchers $[4,6-12,16,17]$. It is worth mentioning that the distributed delay occurs frequently in networks and it has been drawing increasing research attention [6, 16, 17]. Very recently,Liu et al. introduced the distributed delay in the form of constant delay into the discrete-time systems $[9,10]$. However, in practice, time-varying delay in networks occurs commonly in most designs. Therefore, the study of complex networks with distributed time-varying delay is more important than those with constant delays.

In the existing references for complex networks, the deterministic time-delay case was wellstudied; see, for example, $[6,7,9,11,12]$. Actually, the time delay, in such networks exist in a stochastic fashion [18, 29]. In [29], if some values of the time delay in a probabilistic universal learning networks are very large but the probabilities of the delay taking such large values are very small, then it may lead to a more conservative result if only the information of variation range of the time delay is considered. For this case, if one derives the criteria by only using the variation range of time delay, the results could lead to more conservative. In [18], the randomly discrete delay in networks was introduced without considering distributed delay. On the other hand, another interesting random phenomenon, randomly occurring coupling term in complex networks, has been largely overlooked. For networks with communication constraints, such random occurring coupling may be subject to random abrupt changes, which may have resulted from abrupt phenomena such as random failures, changes in the interconnections of subsystems and sudden environment changes, and so forth. An example for randomly occurring coupling is the blackouts in complex bulk power grids in many nations [30], which result in huge losses. To the best of our knowledge, the synchronization problem for discrete-time complex networks with distributed time-varying delay has received very little attention, not to mention the case that randomly occurring coupling and randomly distributed delay are also involved.

Motivated by the above discussions, the aim of this paper is to investigate the synchronization of discrete-time complex networks with randomly occurring coupling and distributed delay in mean square. By using two stochastic variables which satisfy Bernoulli random binary distribution, we propose a new model of complex networks. The effects of both variation range and probability distribution of distributed delay are considered to derive the stability criteria. The proposed results take some well-studied models as special cases. Via a Lyapunov-Krasovskii functional and some new analysis techniques, some sufficient conditions for synchronization in mean square are established for the addressed networks with randomly occurring coupling and distributed delay. An illustrative example is given to show the effectiveness of the proposed results.

\section{Preliminaries}

\section{Notations}

Throughout this paper, $\mathbb{R}^{n}$ and $\mathbb{R}^{n \times m}$ denote, respectively, the $n$-dimensional Euclidean space and the set of all real matrices. The superscript " $T$ " denotes matrix transposition and the notation $X \geq Y$ (resp., $X>Y$ ), where $X$ and $Y$ are symmetric matrices, means that $X-Y$ is positive semidefinite (resp., positive definite). In symmetric block matrices, the symbol $*$ is used as an ellipsis for terms induced by symmetry. $\mathbb{Z}_{\geq 0}$ denotes the set including zero and positive integers. $\mathbb{E}\{x\}$ and $\mathbb{E}\{x \mid y\}$ denote the expectation of $x$ and the expectation of $x$ 
conditional on $y_{.}|\cdot|$ stands for the Euclidean vector norm in $\mathbb{R}^{n}$. The Kronecker product of matrices $Q \in \mathbb{R}^{m \times n}$ and $R \in \mathbb{R}^{p \times q}$ is a matrix in $\mathbb{R}^{m p \times n q}$ and denoted as $Q \otimes R$. coupling:

Consider complex networks with mixed time-varying delays and randomly occurring

$$
\begin{aligned}
x_{i}(k+1)= & f\left(x_{i}(k)\right)+g\left(x_{i}(k-\tau(k))\right)+\sum_{m=-d(k)}^{-1} h\left(x_{i}(k+m)\right)+\rho(k) \sum_{j=1}^{N} w_{1 i j} \Gamma x_{j}(k) \\
& +(1-\rho(k)) \sum_{j=1}^{N} w_{2 i j} \Gamma x_{j}(k), \quad i=1,2, \ldots, N
\end{aligned}
$$

where $x_{i}(k)=\left[x_{i 1}(k), x_{i 2}(k), \ldots, x_{i n}(k)\right]^{T} \in \mathbb{R}^{n}$ is the state vector of the $i$ th node; $f\left(x_{i}(k)\right)=\left(f_{1}\left(x_{i 1}(k)\right), \ldots, f_{n}\left(x_{i n}(k)\right)\right)^{T}, g\left(x_{i}(k)\right)=\left(g_{1}\left(x_{i 1}(k)\right), \ldots, g_{n}\left(x_{i n}(k)\right)\right)^{T}$, and $h\left(x_{i}(k)\right)=\left(h_{1}\left(x_{i 1}(k)\right), \ldots, h_{n}\left(x_{i n}(k)\right)\right)^{T}$ are unknown but sector-bounded nonlinear function; $\tau(k)$ and $d(k)$ denote discrete and distributed time-varying delays, respectively; $\Gamma>0$ represents the inner coupling matrix between the subsystems; $W_{q}=\left(w_{q i j}\right)_{N \times N}$ is the coupling configuration matrices representing the coupling strength and the topological structure of the networks satisfying

$$
w_{q i j}=w_{q j i} \geq 0 \quad(i \neq j), \quad w_{q i i}=\sum_{j=1, j \neq i}^{N} w_{q i j}, \quad q=1,2, i, j=1,2, \ldots, N .
$$

$\rho(k)$ is a stochastic variable that describes the following random events for the system (2.1):

Event 1: system (2.1) experiences $W_{1}$,

Event 2: $\quad$ system (2.1) experiences $W_{2}$.

Let $\rho(k)$ be a Bernoulli distributed sequence defined by

$$
\rho(k)= \begin{cases}1, & \text { if Event } 1 \text { occurs, } \\ 0, & \text { if Event } 2 \text { occurs }\end{cases}
$$

where $\rho(k)$ satisfies $\operatorname{Prob}\{\rho(k)=1\}=\mathbb{E}\{\rho(k)\}=\rho_{0}$, and $\operatorname{Prob}\{\rho(k)=0\}=\bar{\rho}_{0}$.

Remark 2.1. Recently,Liu et al. introduced the distributed delay in discrete-time systems [9, 10]. However, the distributed delay in their well-studied works is considered as constant distributed delay or infinite distributed delay. Due to the fact that time-varying delay takes the usual time delay as special case, in this paper, we aim to investigate synchronization of complex networks with the time-varying distributed delay. In next section, we use some novel techniques to deal with the interval time-varying distributed delay in discrete-time systems.

Remark 2.2. In the existing results, the synchronization of complex networks with deterministic coupling or coupling with stochastic disturbances has been well studied; see, for example, $[4,11,12]$. Different with these works, in this paper, the random variable $\rho(k)$ is used to model 
the probability distribution of the coupling terms. To our knowledge, this represents the first attempt to take into account the occurrence of different coupling terms in a probabilistic way by binary description for the addressed complex networks. In this paper, we call this coupling term as randomly occurring coupling.

Remark 2.3. As mentioned in the introduction, randomly occurring coupling in complex system resides in every field of our world. For example, the blackouts in complex bulk power girds are a case of randomly occurring coupling. In usual time, the connections are normal, which means that $\rho_{0} \gg \bar{\rho}_{0}$. If the blackouts happen in complex bulk power girds, the case of $\rho(k)=0$ occurs and the connections break down.

Throughout this paper, the following assumptions and definitions are needed.

Assumption 2.4. For for all $u, v \in \mathbb{R}^{n}$, the nonlinear function $f(\cdot)$ and $g(\cdot)$ are assumed to satisfy the following sector-bounded condition

$$
\begin{gathered}
\left(f(u)-f(v)-H_{1}(u-v)\right)^{T}\left(f(u)-f(v)-H_{2}(u-v)\right) \leq 0, \\
\left(g(u)-g(v)-M_{1}(u-v)\right)^{T}\left(g(u)-g(v)-M_{2}(u-v)\right) \leq 0, \\
\left(h(u)-h(v)-L_{1}(u-v)\right)^{T}\left(h(u)-h(v)-L_{2}(u-v)\right) \leq 0,
\end{gathered}
$$

where $H_{1}, H_{2}, M_{1}, M_{2}, L_{1}$, and $L_{2}$ are real constant matrices.

Remark 2.5. This assumption was first introduced in [25] and has been subsequently used in many recent networks papers (see, e.g., $[9,11,12,16]$ ). The nonlinear functions in Assumption 2.4 are known as sector-like descriptions of the nonlinearities, which are in a more general form than the usual Lipschitz condition.

Assumption 2.6. The discrete time-varying delay $\tau(k)$ and distributed time-varying delay $d(k)$ are bounded, namely, $0<\tau_{m} \leq \tau(k) \leq \tau_{M}, 0<d_{m} \leq d(k) \leq d_{M}$. And the distributed delay's probability distribution can be observed; that is, assume that $d(k)$ takes values in $\left[d_{1}^{m}: d_{1}^{M}\right]$ or $\left[d_{2}^{m}: d_{2}^{M}\right]$ and $\operatorname{Prob}\left\{d(k) \in\left[d_{1}^{m}: d_{1}^{M}\right]\right\}=\xi_{0}$, where $d_{m} \leq d_{1}^{m}<d_{1}^{M}<d_{2}^{m}<d_{2}^{M} \leq$ $d^{M}$, and $0 \leq \xi_{0} \leq 1$.

Define the two mapping functions

$$
d_{1}(k): \mathbb{Z}_{\geq 0} \longrightarrow\left[d_{1}^{m}: d_{1}^{M}\right], \quad d_{2}(k): \mathbb{Z}_{\geq 0} \longrightarrow\left[d_{2}^{m}: d_{2}^{M}\right],
$$

where for any $k_{0} \in \mathbb{Z}_{\geq 0}$ such that $d_{k_{0}} \in\left[d_{i}^{m}: d_{i}^{M}\right]$, we have $d_{i}^{k_{0}}=d_{k 0}(i=1,2)$. Then, define the two sets

$$
\mathcal{R}_{1}=\left\{k \mid k \in \mathbb{Z}_{\geq 0}, d(k)=d_{1}(k)\right\}, \quad \mathcal{R}_{2}=\left\{k \mid k \in \mathbb{Z}_{\geq 0}, d(k)=d_{2}(k)\right\} .
$$

Clearly, $\mathcal{R}_{1} \cup \mathcal{R}_{2}=\mathbb{Z}_{\geq 0}$ and $\mathcal{R}_{1} \cap \mathcal{R}_{2}=\Phi$ (empty set). Define a stochastic variable as

$$
\xi(k)= \begin{cases}1, & k \in \mathcal{R}_{1} \\ 0, & k \in \mathcal{R}_{2} .\end{cases}
$$


In the following, we denote $x(k), x(k+i), x(k-\tau(k)), \tau(k), d(k), d_{1}(k), d_{2}(k), \rho(k)$, and $\xi(k)$, by $x_{k}, x_{k+i}, x_{\tau}, \tau_{k}, d_{k}, d_{k, 1}, d_{k, 2}, \rho_{k}$, and $\xi_{k}$, respectively. From the above discussion, the complex networks with randomly distributed time-varying delay and coupling can be described in compact form by using Kronecker product " $\otimes$ " of matrices:

$$
\begin{aligned}
x_{k+1}= & F\left(x_{k}\right)+G\left(x_{\tau}\right)+\xi_{k} \sum_{i=-d_{k, 1}}^{-1} H\left(x_{k+i}\right)+\left(1-\xi_{k}\right) \sum_{i=-d_{k, 2}}^{-1} H\left(x_{k+i}\right) \\
& +\rho_{k}\left(W_{1} \otimes \Gamma\right) x_{k}+\left(1-\rho_{k}\right)\left(W_{2} \otimes \Gamma\right) x_{k}
\end{aligned}
$$

where $x_{k}=\left(x_{k, 1}^{T}, x_{k, 2}^{T}, \ldots, x_{k, N}^{T}\right)^{T}, F\left(x_{k}\right)=\left(f^{T}\left(x_{k, 1}\right), f^{T}\left(x_{k, 2}\right), \ldots, f^{T}\left(x_{k, N}\right)\right)^{T}, G\left(x_{\tau}\right)=$ $\left(g^{T}\left(x_{\tau, 1}\right), g^{T}\left(x_{\tau, 2}\right), \ldots, g^{T}\left(x_{\tau, N}\right)\right)^{T}, H\left(x_{k}\right)=\left(h^{T}\left(x_{k, 1}\right), h^{T}\left(x_{k, 2}\right), \ldots, h^{T}\left(x_{k, N}\right)\right)^{T}$.

Remark 2.7. Clearly, in $[9,11,12]$, the variation range of the discrete delay is employed to derive the stability criteria. However, in practical situation, if some values of the delay are very large but the probabilities of the delay taking such large values are very small, it may result in a more conservative result if only the variation range of mixed time delays is considered. By using the new functions $d_{k, 1}, d_{k, 2}, \xi_{k},(2.1)$ can be converted into an equivalent form (2.9). In (2.9), the probabilistic effects of the mixed time delays have been translated into the parameter matrices of the transformed system. Thus, it can be seen that, based on the new model (2.9), the synchronization conditions can be derived.

Remark 2.8. Note that the introduction of time delay in the form of binary stochastic variable was first proposed in [31] and then successfully used in [18, 32, 33]. In [18], the authors employ stochastic discrete time-varying delay to investigate the stability of neural networks. Different with this paper, we consider the randomly distributed time-varying delay in networks. It is also worth mentioning that it is easy to extend our main results to randomly discrete delay in complex networks.

Remark 2.9. From Assumption 2.6 and the definitions of $\rho_{k}$ and $\xi_{k}$, it can be seen that $\rho_{k}$ and $\xi_{k}$ are Bernoulli distributed white sequences with $\operatorname{Prob}\left\{\xi_{k}=1\right\}=\mathbb{E}\left\{\xi_{k}\right\}=\xi_{0}$ and $\operatorname{Prob}\left\{\xi_{k}=\right.$ $0\}=\bar{\xi}_{0}$. Moreover, it can be shown that $\mathbb{E}\left\{\rho_{k}-\rho_{0}\right\}=0, \mathbb{E}\left\{\xi_{k}-\xi_{0}\right\}=0, \mathbb{E}\left\{\left(\rho_{k}-\rho_{0}\right)^{2}\right\}=\rho_{0} \bar{\rho}_{0}$, and $\mathbb{E}\left\{\left(\xi_{k}-\xi_{0}\right)^{2}\right\}=\xi_{0} \bar{\xi}_{0}$.

Definition 2.10. The discrete-time complex networks with randomly distributed time-varying delay and occurring coupling are synchronized in mean square if the following holds:

$$
\lim _{k \rightarrow+\infty} \mathbb{E}\left\{\left|x_{k, i}-x_{k, j}\right|^{2}\right\}=0, \quad \forall 1 \leq i<j \leq N
$$

\section{Main Results}

Before we give the main results of this paper, the following lemmas are needed.

Lemma 3.1. The Kronecker product has the following properties:

(1) $(\alpha A) \otimes B=A \otimes(\alpha B)$,

(2) $(A+B) \otimes C=A \otimes C+B \otimes C$, 
(3) $(A \otimes B)(C \otimes D)=(A C) \otimes(B D)$,

(4) $(A \otimes B)^{T}=A^{T} \otimes B^{T}$.

Lemma 3.2. Let $\mathcal{U}=\left(\alpha_{i j}\right)_{N \times N}, P \in \mathbb{R}^{n \times n}, x=\left(x_{1}^{T}, x_{2}^{T}, \ldots, x_{N}^{T}\right)^{T}$, where $x_{i}=\left(x_{i 1}, x_{i 2}, \ldots, x_{i n}\right)^{T} \in$ $\mathbb{R}^{n}$, and $y=\left(y_{1}^{T}, y_{2}^{T}, \ldots, y_{N}^{T}\right)^{T}$, where $y_{i}=\left(y_{i 1}, y_{i 2}, \ldots, y_{i n}\right)^{T} \in \mathbb{R}^{n}(k=1,2, \ldots, N)$. If $\mathfrak{u}=\mathfrak{u}^{T}$ and each row sum of $\mathfrak{U}$ is zero, then

$$
x^{T}(\mathcal{U} \otimes P) y=-\sum_{1 \leq i<j \leq N} \alpha_{i j}\left(x_{i}-x_{j}\right)^{T} P\left(y_{i}-y_{j}\right)
$$

Lemma 3.3 (see [9]). Let $\mathcal{M} \in \mathbb{R}^{n \times n}$ be a positive semidefinite matrix, let $x_{i} \in \mathbb{R}^{n}$ be a vector, and let $a_{i} \geq 0(i=1,2, \ldots)$ be scalars. If the series concerned are convergent, then the following inequality holds:

$$
\left(\sum_{i=1}^{+\infty} a_{i} x_{i}\right)^{T} \mathcal{M}\left(\sum_{i=1}^{+\infty} a_{i} x_{i}\right) \leq\left(\sum_{i=1}^{+\infty} a_{i}\right) \sum_{i=1}^{+\infty} a_{i} x_{i}^{T} \mathcal{M} x_{i}
$$

Lemma 3.4 (see [9]). Let $A=\left(a_{i j}\right)_{m \times n}, B=\left(b_{i j}\right)_{p \times q}$, and $C=\left(c_{i j}\right)_{m \times q}=A B$. If the sum of all elements in each column of $A$ (row of $B$ ) is zero, then the sum of all elements in each column (row) of $C$ is zero. Moreover, if $A$ is a symmetric matrix and the sum of all elements in each row of $A$ is zero, then, for any positive integer $n$, the sum of all elements in each row of $A^{n}$ is zero.

For the sake of simplicity, we denote by $w_{q i j}^{(2)}, q=1,2$, the $(i, j)$ entry of the matrix $W_{q}^{2}(q=1,2)$. Now, we give our main result in this paper as follows.

Theorem 3.5. Under Assumptions $\left(A_{1}\right)-\left(A_{2}\right)$, the discrete-time complex networks with randomly coupling and distributed time-varying delays are synchronized in mean square, if there exist positive 
definite matrices $P, Q, R_{1}, R_{2}$ and diagonal matrices $V, S, Z$ with appropriate dimensions such that the following matrix inequalities hold:

$$
\begin{aligned}
& \Xi_{i j}=\left[\begin{array}{ccccccc}
\Xi_{i j 11} & \Xi_{i j 12} & S \check{M} & \Xi_{i j 14} & Z \check{L} & \Xi_{i j 16} & \Xi_{i j 17} \\
* & \Xi_{i j 22} & 0 & P & 0 & \xi_{0} P & \bar{\xi}_{0} P \\
* & * & \Xi_{i j 33} & 0 & 0 & 0 & 0 \\
* & * & * & P-Q & 0 & \xi_{0} P & \bar{\xi}_{0} P \\
* & * & * & * & \Xi_{i j 55} & 0 & 0 \\
* & * & * & * & * & \Xi_{i j 66} & 0 \\
* & * & * & * & * & * & \Xi_{i j 77}
\end{array}\right]<0, \quad 1 \leq i<j \leq N \\
& \Xi_{i j 11}=-\rho_{0} N w_{1 i j}^{(2)} \Gamma P \Gamma-\bar{\rho}_{0} N w_{2 i j}^{(2)} \Gamma P \Gamma-P-V \widehat{H}-S \widehat{M}-Z \widehat{L}, \\
& \Xi_{i j 12}=-\rho_{0} N w_{1 i j} P \Gamma-\bar{\rho}_{0} N w_{2 i j} P \Gamma+V \check{H}, \\
& \Xi_{i j 14}=-\rho_{0} N w_{1 i j} P \Gamma-\bar{\rho}_{0} N w_{2 i j} P \Gamma \text {, } \\
& \Xi_{i j 16}=-\xi_{0} \rho_{0} N w_{1 i j} P \Gamma-\xi_{0} \bar{\rho}_{0} N w_{2 i j} P \Gamma, \\
& \Xi_{i j 17}=-\bar{\xi}_{0} \rho_{0} N w_{1 i j} P \Gamma-\bar{\xi}_{0} \bar{\rho}_{0} N w_{2 i j} P \Gamma \text {, } \\
& \Xi_{i j 22}=P-V \text {, } \\
& \Xi_{i j 33}=\left(\tau_{M}-\tau_{m}+1\right) Q-S, \\
& \Xi_{i j 55}=\left[d_{1}^{M}+\frac{1}{2}\left(d_{1}^{M}-d_{1}^{m}\right)\left(d_{1}^{M}+d_{1}^{m}-1\right)\right] R_{1} \\
& +\left[d_{2}^{M}+\frac{1}{2}\left(d_{2}^{M}-d_{2}^{m}\right)\left(d_{2}^{M}+d_{2}^{m}-1\right)\right] R_{2}-Z, \\
& \Xi_{i j 66}=\xi_{0} P-\frac{1}{d_{1}^{M}} R_{1} \text {, } \\
& \Xi_{i j 77}=\bar{\xi}_{0} P-\frac{1}{d_{2}^{M}} R_{2} .
\end{aligned}
$$

Proof. We construct the following Lyapunov-Krasovskii functional $V\left(x_{k}, k\right)$ by

$$
V\left(x_{k}, k\right)=\sum_{i=1}^{4} V_{i}\left(x_{k}, k\right)
$$


where

$$
\begin{aligned}
V_{1}\left(x_{k}, k\right)= & x_{k}^{T}(U \otimes P) x_{k} \\
V_{2}\left(x_{k}, k\right)= & \sum_{i=k-\tau_{k, 1}}^{k-1} G^{T}\left(x_{i}\right)(U \otimes Q) G\left(x_{i}\right)+\sum_{i=-\tau_{M}+1}^{-\tau_{m}} \sum_{j=k+i}^{k-1} G^{T}\left(x_{j}\right)(U \otimes Q) G\left(x_{j}\right), \\
V_{3}\left(x_{k}, k\right)= & \sum_{i=-d_{k, 1}}^{-1} \sum_{j=k+i}^{k-1} H^{T}\left(x_{j}\right)\left(U \otimes R_{1}\right) H\left(x_{j}\right) \\
& +\sum_{i=-d_{1}^{M}}^{-d_{1}^{m}-1} \sum_{j=i+1}^{-1} \sum_{l=k+j}^{k-1} H^{T}\left(x_{l}\right)\left(U \otimes R_{1}\right) H\left(x_{l}\right), \\
V_{4}\left(x_{k}, k\right)= & \sum_{i=-d_{k, 2}}^{-1} \sum_{j=k+i}^{k-1} H^{T}\left(x_{j}\right)\left(U \otimes R_{2}\right) H\left(x_{j}\right) \\
& +\sum_{i=-d_{2}^{M}-1}^{M} \sum_{j=i+1}^{-1} \sum_{l=k+j}^{k-1} H^{T}\left(x_{l}\right)\left(U \otimes R_{2}\right) H\left(x_{l}\right),
\end{aligned}
$$

with

$$
U=\left[\begin{array}{cccc}
N-1 & -1 & \cdots & -1 \\
-1 & N-1 & \cdots & -1 \\
\cdots & \cdots & \cdots & \cdots \\
-1 & -1 & \cdots & N-1
\end{array}\right]_{N \times N}
$$

Calculating the difference of $V\left(x_{k}, k\right)$, we have

$$
\mathbb{E}\left\{\Delta V\left(x_{k}, k\right)\right\}=\sum_{i=1}^{4} \mathbb{E}\left\{\Delta V_{i}\left(x_{k}, k\right)\right\}
$$


where

$$
\begin{aligned}
\mathbb{E}\left\{\Delta V_{1}\left(x_{k}, k\right)\right\}= & \mathbb{E}\left\{V_{1}\left(x_{k+1}, k+1\right) \mid x_{k}\right\}-V_{1}\left(x_{k}, k\right) \\
= & F^{T}\left(x_{k}\right)(U \otimes P) F\left(x_{k}\right)+G^{T}\left(x_{\tau}\right)(U \otimes P) G\left(x_{\tau}\right)+\xi_{0} \sum_{i=-d_{k, 1}}^{-1} H^{T}\left(x_{k+i}\right)(U \otimes P) \\
& \times \sum_{i=-d_{k, 1}}^{-1} H\left(x_{k+i}\right)+\bar{\xi}_{0} \sum_{i=-d_{k, 2}}^{-1} H^{T}\left(x_{k+i}\right)(U \otimes P) \sum_{i=-d_{k, 2}}^{-1} H\left(x_{k+i}\right) \\
& +\rho_{0} x_{k}^{T}\left(W_{1} \otimes \Gamma\right)^{T}(U \otimes P)\left(W_{1} \otimes \Gamma\right) x_{k}+\bar{\rho}_{0} x_{k}^{T}\left(W_{2} \otimes \Gamma\right)^{T}(U \otimes P)\left(W_{2} \otimes \Gamma\right) x_{k} \\
& +2 F^{T}\left(x_{k}\right)(U \otimes P) G\left(x_{\tau}\right)+2 \xi_{0} F^{T}\left(x_{k}\right)(U \otimes P) \sum_{i=-d_{k, 1}}^{-1} H\left(x_{k+i}\right) \\
& +2 \bar{\xi}_{0} F^{T}\left(x_{k}\right)(U \otimes P) \sum_{i=-d_{k, 2}}^{-1} H\left(x_{k+i}\right)+2 \rho_{0} F^{T}\left(x_{k}\right)(U \otimes P)\left(W_{1} \otimes \Gamma\right) x_{k} \\
& +2 \bar{\rho}_{0} F^{T}\left(x_{k}\right)(U \otimes P)\left(W_{2} \otimes \Gamma\right) x_{k}+2 \xi_{0} G^{T}\left(x_{\tau}\right)(U \otimes P) \sum_{i=-d_{k, 1}}^{-1} H\left(x_{k+i}\right) \\
& +2 \bar{\xi}_{0} G^{T}\left(x_{\tau}\right)(U \otimes P) \sum_{i=-d_{k, 2}}^{-1} H\left(x_{k+i}\right)+2 \rho_{0} G^{T}\left(x_{\tau}\right)(U \otimes P)\left(W_{1} \otimes \Gamma\right) x_{k} \\
& +2 \bar{\rho}_{0} G^{T}\left(x_{\tau}\right)(U \otimes P)\left(W_{2} \otimes \Gamma\right) x_{k}+2 \xi_{0} \rho_{0} \sum_{i=-d_{k, 1}}^{-1} H^{T}\left(x_{k+i}\right)(U \otimes P)\left(W_{1} \otimes \Gamma\right) x_{k} \\
& +2 \xi_{0} \bar{\rho}_{0} \sum_{i=-d_{k, 1}}^{-1} H^{T}\left(x_{k+i}\right)(U \otimes P)\left(W_{2} \otimes \Gamma\right) x_{k} \\
& +2 \bar{\xi}_{0} \rho_{0} \sum_{i=-d_{k, 2}}^{-1} H^{T}\left(x_{k+i}\right)(U \otimes P)\left(W_{1} \otimes \Gamma\right) x_{k} \\
& +2 \bar{\xi}_{0} \bar{\rho}_{0} \sum_{i=-d_{k, 2}}^{-1} H^{T}\left(x_{k+i}\right)(U \otimes P)\left(W_{2} \otimes \Gamma\right) x_{k}-x_{k}^{T}(U \otimes P) x_{k}, \\
\mathbb{E}\left\{\Delta V_{2}\left(x_{k}, k\right)\right\}= & \mathbb{E}\left\{V_{2}\left(x_{k+1}, k+1\right) \mid x_{k}\right\}-V_{2}\left(x_{k}, k\right) \\
\leq & 1) G^{T}\left(x_{k}\right)(U \otimes Q) G\left(x_{k}\right)-G^{T}\left(x_{\tau}\right)(U \otimes Q) G\left(x_{\tau}\right) . \\
& \\
& \\
&
\end{aligned}
$$


By using Lemma 3.1, we have

$$
\begin{aligned}
& \mathbb{E}\left\{\Delta V_{3}\left(x_{k}, k\right)\right\}=\mathbb{E}\left\{V_{3}\left(x_{k+1}, k+1\right) \mid x_{k}\right\}-V_{3}\left(x_{k}, k\right) \\
& =\sum_{i=-d_{k+1,1}}^{-1} \sum_{j=k+i+1}^{k} H^{T}\left(x_{j}\right)\left(U \otimes R_{1}\right) H\left(x_{j}\right)-\sum_{i=-d_{k, 1}}^{-1} \sum_{j=k+i}^{k-1} H^{T}\left(x_{j}\right)\left(U \otimes R_{1}\right) H\left(x_{j}\right) \\
& +\sum_{i=-d_{1}^{M}}^{-d_{1}^{m}-1} \sum_{j=i+1}^{-1}\left[\sum_{l=k+j+1}^{k}-\sum_{l=k+j}^{k-1}\right] H^{T}\left(x_{l}\right)\left(U \otimes R_{1}\right) H\left(x_{l}\right) \\
& =\sum_{i=-d_{k+1,1}}^{-1} \sum_{j=k+i+1}^{k-1} H^{T}\left(x_{j}\right)\left(U \otimes R_{1}\right) H\left(x_{j}\right)+\sum_{i=-d_{k+1,1}}^{-1} H^{T}\left(x_{k}\right)\left(U \otimes R_{1}\right) H\left(x_{k}\right) \\
& -\sum_{i=-d_{k, 1}}^{-1} \sum_{j=k+i+1}^{k-1} H^{T}\left(x_{j}\right)\left(U \otimes R_{1}\right) H\left(x_{j}\right)-\sum_{i=k-d_{k, 1}}^{k-1} H^{T}\left(x_{i}\right)\left(U \otimes R_{1}\right) H\left(x_{i}\right) \\
& +\sum_{i=-d_{1}^{M}}^{-d_{1}^{m}-1} \sum_{j=i+1}^{-1}\left[H^{T}\left(x_{k}\right)\left(U \otimes R_{1}\right) H\left(x_{k}\right)-H^{T}\left(x_{k+j}\right)\left(U \otimes R_{1}\right) H\left(x_{k+j}\right)\right] \\
& \leq \sum_{i=-d_{1}^{m}}^{-1} \sum_{j=k+i+1}^{k-1} H^{T}\left(x_{j}\right)\left(U \otimes R_{1}\right) H\left(x_{j}\right)+\sum_{i=-d_{1}^{M}}^{-d_{1}^{m}-1} \sum_{j=k+i+1}^{k-1} H^{T}\left(x_{j}\right)\left(U \otimes R_{1}\right) H\left(x_{j}\right) \\
& +d_{1}^{M} H^{T}\left(x_{k}\right)\left(U \otimes R_{1}\right) H\left(x_{k}\right)-\sum_{i=-d_{1}^{m}}^{-1} \sum_{j=k+i+1}^{k-1} H^{T}\left(x_{j}\right)\left(U \otimes R_{1}\right) H\left(x_{j}\right) \\
& -\sum_{i=-d_{k, 1}}^{-1} H^{T}\left(x_{k+i}\right)\left(U \otimes R_{1}\right) H\left(x_{k+i}\right) \\
& +\frac{1}{2}\left(d_{1}^{M}-d_{1}^{m}\right)\left(d_{1}^{M}+d_{1}^{m}-1\right) H^{T}\left(x_{k}\right)\left(U \otimes R_{1}\right) H\left(x_{k}\right) \\
& -\sum_{i=-d_{1}^{M}}^{-d_{1}^{m}-1} \sum_{j=k+i+1}^{k-1} H^{T}\left(x_{j}\right)\left(U \otimes R_{1}\right) H\left(x_{j}\right) \\
& \leq d_{1}^{M} H^{T}\left(x_{k}\right)\left(U \otimes R_{1}\right) H\left(x_{k}\right)-\sum_{i=-d_{k, 1}}^{-1} H^{T}\left(x_{k+i}\right)\left(U \otimes R_{1}\right) H\left(x_{k+i}\right) \\
& +\frac{1}{2}\left(d_{1}^{M}-d_{1}^{m}\right)\left(d_{1}^{M}+d_{1}^{m}-1\right) H^{T}\left(x_{k}\right)\left(U \otimes R_{1}\right) H\left(x_{k}\right) \quad \text { (Lemma 3.1) } \\
& \leq d_{1}^{M} H^{T}\left(x_{k}\right)\left(U \otimes R_{1}\right) H\left(x_{k}\right) \\
& +\frac{1}{2}\left(d_{1}^{M}-d_{1}^{m}\right)\left(d_{1}^{M}+d_{1}^{m}-1\right) H^{T}\left(x_{k}\right)\left(U \otimes R_{1}\right) H\left(x_{k}\right) \\
& -\frac{1}{d_{1}^{M}}\left(\sum_{i=-d_{k, 1}}^{-1} H\left(x_{k+i}\right)\right)^{T}\left(U \otimes R_{1}\right)\left(\sum_{i=-d_{k, 1}}^{-1} H\left(x_{k+i}\right)\right) \text {. }
\end{aligned}
$$


Similarly, we have

$$
\begin{aligned}
\mathbb{E}\left\{\Delta V_{4}\left(x_{k}, k\right)\right\} \leq & d_{2}^{M} H^{T}\left(x_{k}\right)\left(U \otimes R_{2}\right) H\left(x_{k}\right) \\
& +\frac{1}{2}\left(d_{2}^{M}-d_{2}^{m}\right)\left(d_{2}^{M}+d_{2}^{m}-1\right) H^{T}\left(x_{k}\right)\left(U \otimes R_{2}\right) H\left(x_{k}\right) \\
& -\frac{1}{d_{2}^{M}}\left(\sum_{i=-d_{k, 2}}^{-1} H\left(x_{k+i}\right)\right)^{T}\left(U \otimes R_{2}\right)\left(\sum_{i=-d_{k, 2}}^{-1} H\left(x_{k+i}\right)\right) .
\end{aligned}
$$

Noting that $U W_{i}=W_{i} U=N W_{i}(i=1,2)$, for any matrix $H$, with appropriate dimension, we have from Lemma 3.1 that

$$
\begin{gathered}
(U \otimes H)\left(W_{i} \otimes \Gamma\right)=\left(U W_{i}\right) \otimes\left(H \Gamma_{i}\right)=\left(N W_{i}\right) \otimes(H \Gamma), \\
\left(W_{i} \otimes \Gamma\right)^{T}(U \otimes H)\left(W_{i} \otimes \Gamma\right)=\left(W_{i}^{T} \otimes \Gamma^{T}\right)(U \otimes H)\left(W_{i} \otimes \Gamma\right) \\
=\left(W_{i}^{T} U W_{i}\right) \otimes\left(\Gamma^{T} H \Gamma\right) \\
=\left(N W_{i}^{2}\right) \otimes(\Gamma H \Gamma) .
\end{gathered}
$$

Let $\mathbf{x}_{k, i j}=x_{k, i}-x_{k, j}, \mathbf{f}_{k, i j}=f\left(x_{k, i}\right)-f\left(x_{k, j}\right), \mathbf{g}_{k, i j}=g\left(x_{k, i}\right)-g\left(x_{k, j}\right), \hat{\mathbf{g}}_{k, i j}=g\left(x_{\tau, i}\right)-$ $g\left(x_{\tau, j}\right), \mathbf{h}_{k, i j}=h\left(x_{k, i}\right)-h\left(x_{k, j}\right), \widehat{\mathbf{h}}_{k, i j}=\left(\sum_{m=-d_{k, 1}}^{-1} h\left(x_{k+m, i}\right)\right)-\left(\sum_{m=-d_{k, 1}}^{-1} h\left(x_{k+m, j}\right)\right)$, and $\widetilde{\mathbf{h}}_{k, i j}=$ $\left(\sum_{m=-d_{k, 2}}^{-1} h\left(x_{k+m, i}\right)\right)-\left(\sum_{m=-d_{k, 2}}^{-1} h\left(x_{k+m, j}\right)\right)$.

Combining (3.9)-(3.12) into (3.8) and using Lemmas 3.2 and 3.4, we have

$$
\begin{aligned}
& \mathbb{E}\{\Delta V(k)\} \leq \sum_{1 \leq i<j \leq N}\left\{\mathbf{f}_{k, i j}^{T} P \mathbf{f}_{k, i j}+\widehat{\mathbf{g}}_{k, i j}^{T} P \widehat{\mathbf{g}}_{k, i j}+\xi_{0} \widehat{\mathbf{h}}_{k, i j}^{T} P \widehat{\mathbf{h}}_{k, i j}+\bar{\xi}_{0} \widetilde{\mathbf{h}}_{k, i j}^{T} P \widetilde{\mathbf{h}}_{k, i j}\right. \\
&-\rho_{0} \mathbf{x}_{k, i j}^{T}\left(N w_{1 i j}^{(2)} \Gamma P \Gamma\right) \mathbf{x}_{k, i j}-\bar{\rho}_{0} \mathbf{x}_{k, i j}^{T}\left(N w_{2 i j}^{(2)} \Gamma P \Gamma\right) \mathbf{x}_{k, i j}+2 \mathbf{f}_{k, i j}^{T} P \widehat{\mathbf{g}}_{k, i j} \\
&+2 \xi_{0} \mathbf{f}_{k, i j}^{T} P \widehat{\mathbf{h}}_{k, i j}+2 \bar{\xi}_{0} \mathbf{f}_{k, i j}^{T} P \widetilde{\mathbf{h}}_{k, i j}-2 \rho_{0} \mathbf{f}_{k, i j}^{T}\left(N w_{1 i j} P \Gamma\right) \mathbf{x}_{k, i j} \\
&-2 \bar{\rho}_{0} \mathbf{f}_{k, i j}^{T}\left(N w_{2 i j} P \Gamma\right) \mathbf{x}_{k, i j}+2 \xi_{0} \widehat{\mathbf{g}}_{k, i j}^{T} P \widehat{\mathbf{h}}_{k, i j}+2 \bar{\xi}_{0} \widehat{\mathbf{g}}_{k, i j}^{T} P \widetilde{\mathbf{h}}_{k, i j} \\
&-2 \rho_{0} \widehat{\mathbf{g}}_{k, i j}^{T}\left(N w_{1 i j} P \Gamma\right) \mathbf{x}_{k, i j}-2 \bar{\rho}_{0} \widehat{\mathbf{g}}_{k, i j}^{T}\left(N w_{2 i j} P \Gamma\right) \mathbf{x}_{k, i j} \\
&-2 \xi_{0} \rho_{0} \widehat{\mathbf{h}}_{k, i j}^{T}\left(N w_{1 i j} P \Gamma\right) \mathbf{x}_{k, i j}-2 \xi_{0} \bar{\rho}_{0} \widehat{\mathbf{h}}_{k, i j}^{T}\left(N w_{2 i j} P \Gamma\right) \mathbf{x}_{k, i j} \\
&-2 \bar{\xi}_{0} \rho_{0} \widetilde{\mathbf{h}}_{k, i j}^{T}\left(N w_{1 i j} P \Gamma\right) \mathbf{x}_{k, i j}-2 \bar{\xi}_{0} \bar{\rho}_{0} \widetilde{\mathbf{h}}_{k, i j}^{T}\left(N w_{2 i j} P \Gamma\right) \mathbf{x}_{k, i j} \\
&+\left(\tau_{M}-\tau_{m}+1\right) \mathbf{g}_{k, i j}^{T} Q \mathbf{g}_{k, i j}-\widehat{\mathbf{g}}_{k, i j}^{T} Q \widehat{\mathbf{g}}_{k, i j} \\
&+\left[d_{1}^{M}+\frac{1}{2}\left(d_{1}^{M}-d_{1}^{m}\right)\left(d_{1}^{M}+d_{1}^{m}-1\right)\right] \mathbf{h}_{k, i j}^{T} R_{1} \mathbf{h}_{k, i j}-\frac{1}{d_{1}^{M}} \widehat{\mathbf{h}}_{k, i j}^{T} R_{1} \widehat{\mathbf{h}}_{k, i j} \\
&+\left[d_{2}^{M}+\frac{1}{2}\left(d_{2}^{M}-d_{2}^{m}\right)\left(d_{2}^{M}+d_{2}^{m}-1\right)\right] \mathbf{h}_{k, i j}^{T} R_{2} \mathbf{h}_{k, i j} \\
&\left.-\frac{1}{d_{2}^{M}} \widetilde{\mathbf{h}}_{k, i j}^{T} R_{2} \widetilde{\mathbf{h}}_{k, i j}-\mathbf{x}_{k, i j}^{T} P \mathbf{x}_{k, i j}\right\} .
\end{aligned}
$$


Then, one can derive from $\left(A_{2}\right)$ that

$$
\left[\begin{array}{l}
\mathbf{x}_{k, i j} \\
\mathbf{f}_{k, i j}
\end{array}\right]^{T}\left[\begin{array}{cc}
-V \widehat{H} & V \check{H} \\
V \check{H}^{T} & -V I
\end{array}\right]\left[\begin{array}{l}
\mathbf{x}_{k, i j} \\
\mathbf{f}_{k, i j}
\end{array}\right]+\left[\begin{array}{l}
\mathbf{x}_{k, i j} \\
\mathbf{g}_{k, i j}
\end{array}\right]^{T}\left[\begin{array}{cc}
-S \widehat{M} & S \check{M} \\
S \check{M}^{T} & -S I
\end{array}\right]\left[\begin{array}{l}
\mathbf{x}_{k, i j} \\
\mathbf{g}_{k, i j}
\end{array}\right]+\left[\begin{array}{c}
\mathbf{x}_{k, i j} \\
\mathbf{h}_{k, i j}
\end{array}\right]^{T}\left[\begin{array}{cc}
-Z \widehat{L} & Z \check{L} \\
Z \check{L}^{T} & -Z I
\end{array}\right]\left[\begin{array}{l}
\mathbf{x}_{k, i j} \\
\mathbf{h}_{k, i j}
\end{array}\right] \geq 0,
$$

with $\widehat{H}=\left(H_{1}^{T} H_{2}+H_{2}^{T} H_{1}\right) / 2, \check{H}=\left(H_{1}^{T}+H_{2}^{T}\right) / 2, \widehat{M}=\left(M_{1}^{T} M_{2}+M_{2}^{T} M_{1}\right) / 2, \check{M}=\left(M_{1}^{T}+\right.$ $\left.M_{2}^{T}\right) / 2, \widehat{L}=\left(L_{1}^{T} L_{2}+L_{2}^{T} L_{1}\right) / 2$, and $\breve{L}=\left(L_{1}^{T}+L_{2}^{T}\right) / 2$.

Using (3.13) and (3.14), we obtain

$$
\mathbb{E}\{\Delta V(k)\} \leq \sum_{1 \leq i<j \leq N} \eta_{i j}^{T}(k) \Xi_{i j} \eta_{i j}(k)
$$

where $\eta_{i j}(k)=\left[\mathbf{x}_{k, i j}^{T} \mathbf{f}_{k, i j}^{T}, \mathbf{g}_{k, i j}^{T}, \widehat{\mathbf{g}}_{k, i j}^{T}, \mathbf{h}_{k, i j}^{T}, \widehat{\mathbf{h}}_{k, i j}^{T}, \tilde{\mathbf{h}}_{k, i j}^{T}\right]^{T}$ and $\Xi_{i j}$ is defined in (3.3). It follows that

$$
\mathbb{E}\{\Delta V(k)\} \leq \lambda_{\max }\left(\Xi_{i j}\right) \sum_{1 \leq i<j \leq N}\left|\eta_{i j}(k)\right|^{2} .
$$

Note that $\lambda_{\max }\left(\Xi_{i j}\right)<0$ and let $\lambda_{0}=\max _{1 \leq i<j \leq N}\left(\lambda_{\max }\left(\Xi_{i j}\right)\right)$. We have $\lambda_{0}<0$, and then, it follows readily from (3.16) that

$$
\mathbb{E}\{\Delta V(k)\} \leq \lambda_{0} \mathbb{E}\left\{\sum_{1 \leq i<j \leq N}\left|\mathbf{x}_{k, i j}\right|^{2}\right\} .
$$

Letting $m$ be a positive integer, the summation of both sides of (3.17) from 1 to $m$ with respect to $k$ yields

$$
\mathbb{E}\{\Delta V(k)\} \leq \lambda_{0} \sum_{1 \leq i<j \leq N} \sum_{k=1}^{m} \mathbb{E}\left\{\left|\mathbf{x}_{k, i j}\right|^{2}\right\}
$$

which implies that

$$
-\lambda_{0} \sum_{1 \leq i<j \leq N} \sum_{k=1}^{m} \mathbb{E}\left\{\left|\mathbf{x}_{k, i j}\right|^{2}\right\} \leq \mathbb{E}\{V(1)\}
$$

It is can be concluded that the series $\sum_{k=1}^{+\infty} \mathbb{E}\left\{\left|\mathbf{x}_{k, i j}\right|^{2}\right\}$ is convergent, and therefore

$$
\lim _{k \rightarrow+\infty} \mathbb{E}\left\{\left|x_{k, i}-x_{k, j}\right|^{2}\right\}=\lim _{k \rightarrow+\infty} \mathbb{E}\left\{\left|\mathbf{x}_{k, i j}\right|^{2}\right\}=0
$$

This completes the proof of the theorem. 
Remark 3.6. If the distributed time-varying delay reduces to infinite distributed delay and binary descriptions were removed from (3.5), the model reduces to model studied in [9], and then the results obtained in this paper are equivalent to those of Theorem 1 in [9]. On the other hand, the authors studied the stability problem in neural networks with randomly discrete delay [18]. It is worth mentioning that we can easily extend our results to the case of stability problem of neural networks with randomly distributed delay.

Remark 3.7. In $[6,7,9]$, the synchronization problem of complex networks with deterministic coupling has been studied. In practical situations, the coupling in networks may be subject to some noise. In $[4,11,12]$, synchronization of complex networks with stochastic disturbances has been well studied. However, another interesting phenomenon, randomly occurring coupling term in complex networks, has been largely overlooked. In this paper, we introduce a Bernoulli stochastic variable to describe the stochastic occurring coupling.

If the randomly occurring coupling does not happen in complex networks, that is, $\rho(k)=1$, then we can obtain the following results.

Corollary 3.8. Under Assumptions $\left(A_{1}\right)-\left(A_{2}\right)$, the discrete-time complex networks with randomly distributed time-varying delays are synchronized in mean square, if there exist positive definite matrices $P, Q, R_{1}, R_{2}$ and diagonal matrices $V, S, Z$ with appropriate dimensions such that the following matrix inequalities hold:

$$
\begin{aligned}
\Xi_{i j}= & {\left[\begin{array}{ccccccc}
\Xi_{i j 11} & \Xi_{i j 12} & S \check{M} & \Xi_{i j 14} & Z \breve{L} & \Xi_{i j 16} & \Xi_{i j 17} \\
* & \Xi_{i j 22} & 0 & P & 0 & \xi_{0} P & \bar{\xi}_{0} P \\
* & * & \Xi_{i j 33} & 0 & 0 & 0 & 0 \\
* & * & * & P-Q & 0 & \xi_{0} P & \bar{\xi}_{0} P \\
* & * & * & * & \Xi_{i j 55} & 0 & 0 \\
* & * & * & * & * & \Xi_{i j 66} & 0 \\
* & * & * & * & * & * & \Xi_{i j 77}
\end{array}\right]<0, \quad 1 \leq i<j \leq N, } \\
\Xi_{i j 11}= & -N w_{1 i j}^{(2)} \Gamma P \Gamma-P-V \widehat{H}-S \widehat{M}-Z \widehat{L}, \\
\Xi_{i j 12}= & -N w_{1 i j} P \Gamma+V \breve{H}, \\
\Xi_{i j 14}= & -N w_{1 i j} P \Gamma, \\
\Xi_{i j 16}= & -\xi_{0} N w_{1 i j} P \Gamma, \\
\Xi_{i j 17}= & -\bar{\xi}_{0} N w_{1 i j} P \Gamma, \\
\Xi_{i j 22}= & P-V, \\
\Xi_{i j 33}= & \left(\tau_{M}-\tau_{m}+1\right) Q-S, \\
\Xi_{i j 55}= & {\left[d_{1}^{M}+\frac{1}{2}\left(d_{1}^{M}-d_{1}^{m}\right)\left(d_{1}^{M}+d_{1}^{m}-1\right)\right] R_{1} } \\
& +\left[d_{2}^{M}+\frac{1}{2}\left(d_{2}^{M}-d_{2}^{m}\right)\left(d_{2}^{M}+d_{2}^{m}-1\right)\right] R_{2}-Z, \\
\Xi_{i j 66}= & \xi_{0} P-\frac{1}{d_{1}^{M}} R_{1}, \\
\Xi_{i j 77=}= & \bar{\xi}_{0} P-\frac{1}{d_{2}^{M}} R_{2} .
\end{aligned}
$$


If the distributed time-varying delays do not occur stochastically, that is, $\xi(k)=1$, then we have the following results.

Corollary 3.9. Under assumptions $\left(A_{1}\right)-\left(A_{2}\right)$, the discrete-time complex networks with distributed time-varying delays is synchronized in mean square, if there exist positive definite matrices $P, Q, R_{1}$ and diagonal matrices $V, S, Z$ with appropriate dimensions such that the following matrix inequalities hold:

$$
\begin{aligned}
\Xi_{i j}= & {\left[\begin{array}{cccccc}
\Xi_{i j 11} & \Xi_{i j 12} & S \check{M} & \Xi_{i j 14} & Z \check{L} & \Xi_{i j 16} \\
* & \Xi_{i j 22} & 0 & P & 0 & P \\
* & * & \Xi_{i j 33} & 0 & 0 & 0 \\
* & * & * & P-Q & 0 & P \\
* & * & * & * & \Xi_{i j 55} & 0 \\
* & * & * & * & * & \Xi_{i j 66}
\end{array}\right]<0, \quad 1 \leq i<j \leq N, } \\
\Xi_{i j 11} & =-\rho_{0} N w_{1 i j}^{(2)} \Gamma P \Gamma-\bar{\rho}_{0} N w_{2 i j}^{(2)} \Gamma P \Gamma-P-V \widehat{H}-S \widehat{M}-Z \widehat{L}, \\
\Xi_{i j 12} & =-\rho_{0} N w_{1 i j} P \Gamma-\bar{\rho}_{0} N w_{2 i j} P \Gamma+V \check{H}, \\
\Xi_{i j 14} & =-\rho_{0} N w_{1 i j} P \Gamma-\bar{\rho}_{0} N w_{2 i j} P \Gamma, \\
\Xi_{i j 16} & =-\rho_{0} N w_{1 i j} P \Gamma-\bar{\rho}_{0} N w_{2 i j} P \Gamma, \\
\Xi_{i j 22} & =P-V, \\
\Xi_{i j 33} & =\left(\tau_{M}-\tau_{m}+1\right) Q-S, \\
\Xi_{i j 55} & =\left[d_{1}^{M}+\frac{1}{2}\left(d_{1}^{M}-d_{1}^{m}\right)\left(d_{1}^{M}+d_{1}^{m}-1\right)\right] R_{1}-Z, \\
\Xi_{i j 66} & =P-\frac{1}{d_{1}^{M}} R_{1} .
\end{aligned}
$$

If both coupling and distributed time-varying delays do not occur randomly, that is, $\xi(k)=1$ and $\rho(k)=1$, then we have the following results.

Corollary 3.10. Under Assumptions $\left(A_{1}\right)-\left(A_{2}\right)$, the discrete-time complex networks with distributed time-varying delays are synchronized in mean square, if there exist positive definite matrices $P, Q, R_{1}$ 
and diagonal matrices $V, S, Z$ with appropriate dimensions such that the following matrix inequalities hold:

$$
\begin{aligned}
& =\left[\begin{array}{cccccc}
\Xi_{i j 11} & \Xi_{i j 12} & S \check{M} & \Xi_{i j 14} & Z \check{L} & \Xi_{i j 16} \\
* & \Xi_{i j 22} & 0 & P & 0 & P \\
* & * & \Xi_{i j 33} & 0 & 0 & 0 \\
* & * & * & P-Q & 0 & P \\
* & * & * & * & \Xi_{i j 55} & 0 \\
* & * & * & * & * & \Xi_{i j 66}
\end{array}\right]<0, \quad 1 \leq i<j \leq N, \\
\Xi_{i j} & =-N w_{1 i j}^{(2)} \Gamma P \Gamma-P-V \widehat{H}-S \widehat{M}-Z \widehat{L}, \\
\Xi_{i j 11} & =-N w_{1 i j} P \Gamma+V \check{H}, \\
\Xi_{i j 12} & =-N \Gamma, \\
\Xi_{i j 14} & =-N w_{1 i j} P \Gamma, \\
\Xi_{i j 16} & =-N w_{1 i j} P \Gamma, \\
\Xi_{i j 22} & =P-V, \\
\Xi_{i j 33} & =\left(\tau_{M}-\tau_{m}+1\right) Q-S, \\
\Xi_{i j 55} & =\left[d_{1}^{M}+\frac{1}{2}\left(d_{1}^{M}-d_{1}^{m}\right)\left(d_{1}^{M}+d_{1}^{m}-1\right)\right] R_{1}-Z, \\
\Xi_{i j 66} & =P-\frac{1}{d_{1}^{M}} R_{1} .
\end{aligned}
$$

\section{Numerical Simulation}

We consider the following discrete-time complex networks with randomly coupling and distributed time-varying delay consisting of three nodes:

$$
\begin{aligned}
x_{i}(k+1)= & f\left(x_{i}(k)\right)+g\left(x_{i}(k-\tau(k))\right)+\xi(k) \sum_{m=-d_{1}(k)}^{-1} h\left(x_{i}(k+m)\right) \\
& +(1-\xi(k)) \sum_{m=-d_{2}(k)}^{-1} h\left(x_{i}(k+m)\right)+\rho(k) \sum_{j=1}^{N} w_{1 i j} \Gamma x_{j}(k) \\
& +(1-\rho(k)) \sum_{j=1}^{N} w_{2 i j} \Gamma x_{j}(k), \quad i=1,2, \ldots, N,
\end{aligned}
$$

with $x_{i}(k)=\left(x_{i 1}(k), x_{i 2}(k)\right)^{T}(i=1,2,3)$ being the state vector of the $i$ th subsystem. The discrete delay is chosen as $\tau(k)=3+\left(1+(-1)^{k}\right)$. It can be verified that $\tau_{m}=3, \tau_{M}=5$. We select $\rho_{0}=0.6, \xi_{0}=0.8, d_{1}^{m}=1, d_{1}^{M}=3, d_{2}^{m}=4$, and $d_{2}^{M}=10$.

$$
W_{1}=\left(\begin{array}{ccc}
-0.2 & 0.1 & 0.1 \\
0.1 & -0.2 & 0.1 \\
0.1 & 0.1 & -0.2
\end{array}\right), \quad W_{2}=\left(\begin{array}{ccc}
-0.3 & 0.15 & 0.15 \\
0.15 & -0.3 & 0.15 \\
0.15 & 0.15 & -0.3
\end{array}\right), \quad \Gamma=\left(\begin{array}{cc}
0.5 & 0 \\
0 & 0.5
\end{array}\right)
$$




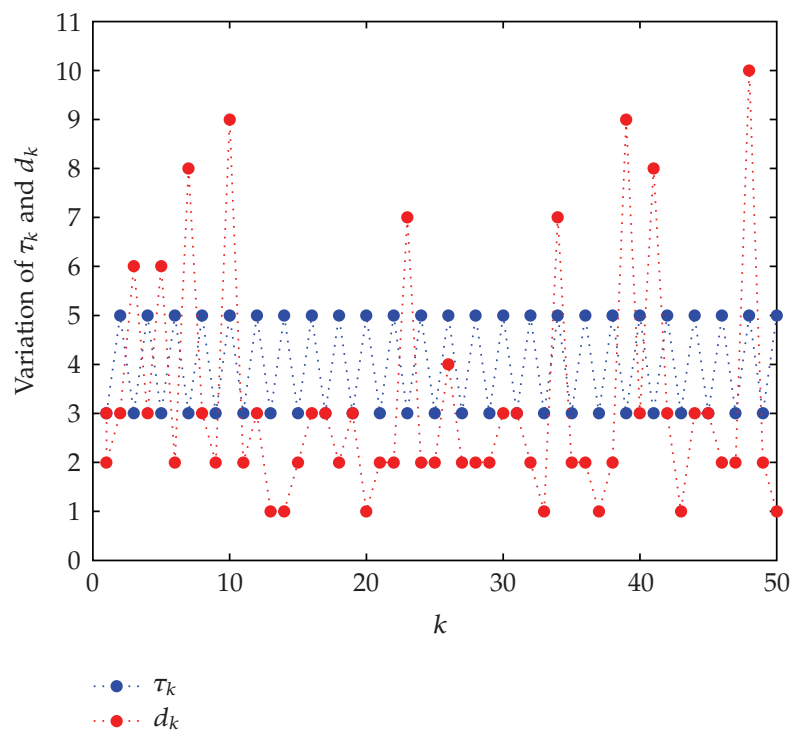

Figure 1: Variation of $\tau_{k}$ and $d_{k}$.

Let the nonlinear vector-valued functions be given by

$$
\begin{gathered}
f\left(x_{i}(k)\right)=\left(-0.5 x_{i 1}(k)+\tanh \left(0.2 x_{i 1}(k)\right)+0.2 x_{i 2}(k), 0.95 x_{i 2}(k)-\tanh \left(0.75 x_{i 2}(k)\right)\right)^{T}, \\
g\left(x_{i}(k)\right)=h\left(x_{i}(k)\right)=\left(0.2 x_{i 1}(k)-\tanh \left(0.1 x_{i 1}(k)\right), 0.1 x_{i 2}(k)\right)^{T}, \quad i=1,2,3 .
\end{gathered}
$$

It can be verified that

$$
\begin{array}{rlrl}
H_{1} & =\left(\begin{array}{cc}
-0.5 & 0.2 \\
0 & 0.95
\end{array}\right), & H_{2} & =\left(\begin{array}{cc}
-0.3 & 0.2 \\
0 & 0.2
\end{array}\right) \\
M_{1}=L_{1} & =\left(\begin{array}{cc}
0.2 & 0 \\
0 & 0.1
\end{array}\right), & M_{2}=L_{2}=\left(\begin{array}{cc}
0.1 & 0 \\
0 & 0.1
\end{array}\right) .
\end{array}
$$

We can obtain LMI solution in the following:

$$
\begin{gathered}
P=\left(\begin{array}{ll}
0.9268 & 0.0011 \\
0.0011 & 0.9188
\end{array}\right), \quad Q=\left(\begin{array}{cc}
62.4010 & 0.0334 \\
0.0334 & 60.4689
\end{array}\right), \quad R_{1}=\left(\begin{array}{ll}
37.5891 & -0.0266 \\
-0.0266 & 37.7721
\end{array}\right), \\
R_{2}=\left(\begin{array}{ll}
6.0396 & 0.0244 \\
0.0244 & 5.7941
\end{array}\right), \quad V=\left(\begin{array}{cc}
81.5029 & 0 \\
0 & 94.2456
\end{array}\right), \\
S=\left(\begin{array}{cc}
270.1472 & 0 \\
0 & 262.9662
\end{array}\right), \quad Z=\left(\begin{array}{cc}
619.6532 & 0 \\
0 & 595.8848
\end{array}\right) .
\end{gathered}
$$

It is clear that the condition of Theorem 3.5 is satisfied. Therefore, the synchronization of complex networks (2.9) can be achieved. For this example, all the stability criteria in $[9,11,12]$ fail to draw a conclusion. This is because, by applying to this example, the LMI conditions in $[9,11,12]$ cannot generate a feasible solution since the distributed delay is timevarying, as 


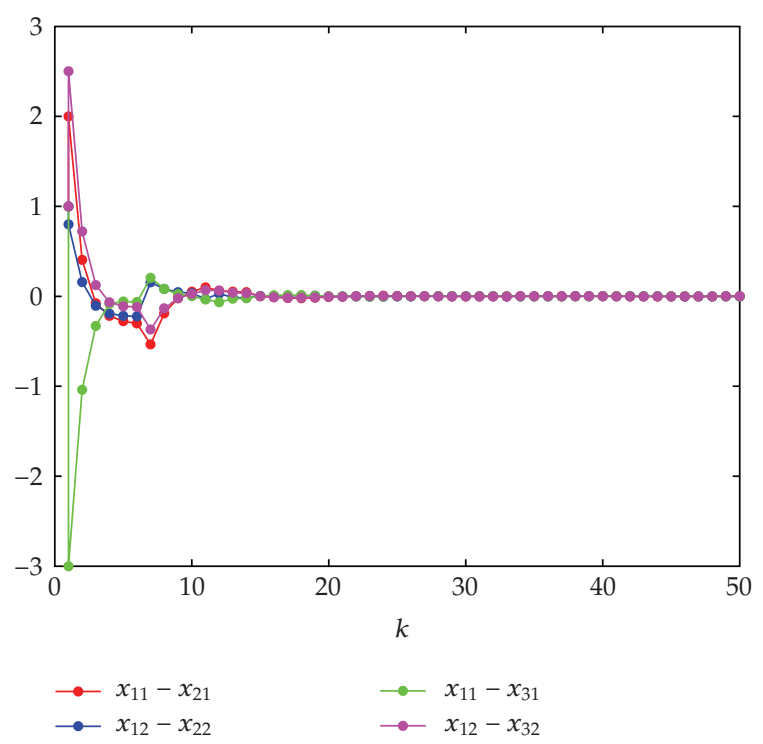

Figure 2: Synchronization errors.

well as the coupling term and distributed delay occur randomly. However, using our criterion in Theorem 3.5, we conclude that the complex networks with randomly occurring coupling and distributed time-varying delays (2.9) can be uniformly synchronized in mean square since the LMI in (3.3) is feasible using LMI Toolbox.

Figure 1 shows the variation of $\tau_{k}$ and $d_{k}$. It can be observed from Figure 2 that the discrete-time complex networks with randomly occurring coupling and stochastic distributed time-varying delay can be synchronized in mean square.

\section{Conclusion}

In this paper, we have investigated the synchronization of novel discrete-time complex networks with randomly occurring coupling and distributed time-varying delay. By employing some new techniques, the Kronecker product and linear matrix inequality (LMI) techniques, the synchronization criteria have been established for discrete-time complex networks with randomly distributed time-varying delay, as well as randomly occurring coupling. In the end of this paper, we have exploited a numerical example to show the usefulness of our results.

\section{Acknowledgments}

This research was partially supported by the National Natural Science Foundation of China (Grant no. 60874113), the Research Fund for the Doctoral Program of Higher Education (Grant no. 200802550007), the Key Creative Project of Shanghai Education Community (Grant no. 09ZZ66), and the Key Foundation Project of Shanghai (Grant no. 09JC1400700). 


\section{References}

[1] C. W. Wu and L. O. Chua, "Synchronization in an array of linearly coupled dynamical systems," IEEE Transactions on Circuits and Systems I, vol. 42, no. 8, pp. 430-447, 1995.

[2] X. F. Wang and G. Chen, "Synchronization in small-world dynamical networks," International Journal of Bifurcation and Chaos, vol. 12, no. 1, pp. 187-192, 2002.

[3] J. Lü and G. Chen, "A time-varying complex dynamical network model and its controlled synchronization criteria," IEEE Transactions on Automatic Control, vol. 50, no. 6, pp. 841-846, 2005.

[4] J. Cao, Z. Wang, and Y. Sun, "Synchronization in an array of linearly stochastically coupled networks with time delays," Physica A, vol. 385, no. 2, pp. 718-728, 2007.

[5] J. Cao, P. Li, and W. Wang, "Global synchronization in arrays of delayed neural networks with constant and delayed coupling," Physics Letters A, vol. 353, no. 4, pp. 318-325, 2006.

[6] J. Cao, G. Chen, and P. Li, “Global synchronization in an array of delayed neural networks with hybrid coupling," IEEE Transactions on Systems, Man, and Cybernetics, Part B, vol. 38, no. 2, pp. 488-498, 2008.

[7] W. Yu, J. Cao, and J. Lü, "Global synchronization of linearly hybrid coupled networks with timevarying delay," SIAM Journal on Applied Dynamical Systems, vol. 7, no. 1, pp. 108-133, 2008.

[8] H. Gao, J. Lam, and G. Chen, "New criteria for synchronization stability of general complex dynamical networks with coupling delays," Physics Letters A, vol. 360, no. 2, pp. 263-273, 2006.

[9] Y. Liu, Z. Wang, J. Liang, and X. Liu, "Synchronization and state estimation for discrete-time complex networks with distributed delays," IEEE Transactions on Systems, Man, and Cybernetics, Part B, vol. 38, no. 5, pp. 1314-1325, 2008.

[10] Y. Liu, Z. Wang, and X. Liu, "State estimation for discrete-time Markovian jumping neural networks with mixed mode-dependent delays," Physics Letters A, vol. 372, no. 48, pp. 7147-7155, 2008.

[11] J. Liang, Z. Wang, Y. Liu, and X. Liu, "Global synchronization control of general delayed discretetime networks with stochastic coupling and disturbances," IEEE Transactions on Systems, Man, and Cybernetics, Part B, vol. 38, no. 4, pp. 1073-1083, 2008.

[12] J. Liang, Z. Wang, Y. Liu, and X. Liu, "Robust synchronization of an array of coupled stochastic discrete-time delayed neural networks," IEEE Transactions on Neural Networks, vol. 19, no. 11, pp. 1910-1921, 2008.

[13] W. Lu and T. Chen, "Synchronization analysis of linearly coupled networks of discrete time systems," Physica D, vol. 198, no. 1-2, pp. 148-168, 2004.

[14] W. Lu and T. Chen, "Global synchronization of discrete-time dynamical network with a directed graph," IEEE Transactions on Circuits and Systems II, vol. 54, no. 2, pp. 136-140, 2007.

[15] Y. Tang, Z. Wang, and J.-A. Fang, "Pinning control of fractional-order weighted complex networks," Chaos, vol. 19, no. 1, Article ID 013112, 9 pages, 2009.

[16] Y. Tang, J.-A. Fang, and Q. Miao, "On the exponential synchronization of stochastic jumping chaotic neural networks with mixed delays and sector-bounded non-linearities," Neurocomputing, vol. 72, no. 7-9, pp. 1694-1701, 2009.

[17] Y. Tang, R. Qiu, J.-A. Fang, Q. Miao, and M. Xia, “Adaptive lag synchronization in unknown stochastic chaotic neural networks with discrete and distributed time-varying delays," Physics Letters A, vol.372, no. 24, pp. 4425-4433, 2008.

[18] D. Yue, Y. Zhang, E. Tian, and C. Peng, “Delay-distribution-dependent exponential stability criteria for discrete-time recurrent neural networks with stochastic delay," IEEE Transactions on Neural Networks, vol. 19, no. 7, pp. 1299-1306, 2008.

[19] S. Xu and J. Lam, "A new approach to exponential stability analysis of neural networks with timevarying delays," Neural Networks, vol. 19, no. 1, pp. 76-83, 2006.

[20] Y. Zhang, D. Yue, and E. Tian, "Robust delay-distribution-dependent stability of discrete-time stochastic neural networks with time-varying delay," Neurocomputing, vol. 72, no. 4-6, pp. 1265-1273, 2009.

[21] Y. He, Q.-G. Wang, M. Wu, and C. Lin, "Delay-dependent state estimation for delayed neural networks," IEEE Transactions on Neural Networks, vol. 17, no. 4, pp. 1077-1081, 2006.

[22] W.-H. Chen, X. Lu, and D.-Y. Liang, "Global exponential stability for discrete-time neural networks with variable delays," Physics Letters A, vol. 358, no. 3, pp. 186-198, 2006.

[23] C. Posadas-Castillo, C. Cruz-Hernández, and R. M. López-Gutiérrez, "Synchronization of chaotic neural networks with delay in irregular networks," Applied Mathematics and Computation, vol. 205, no. 1, pp. 487-496, 2008. 
[24] O. M. Kwon and Ju H. Park, "Exponential stability analysis for uncertain neural networks with interval time-varying delays," Applied Mathematics and Computation, vol. 212, no. 2, pp. 530-541, 2009.

[25] Y. Liu, Z. Wang, and X. Liu, "Global exponential stability of generalized recurrent neural networks with discrete and distributed delays," Neural Networks, vol. 19, no. 5, pp. 667-675, 2006.

[26] V. Singh, "Improved global robust stability of interval delayed neutral networks via split interval: genralizations," Applied Mathematics and Computation, vol. 206, no. 1, pp. 290-297, 2008.

[27] R. Samli and S. Arik, "New results for global stability of a class of neutral-type neural systems with time delays," Applied Mathematics and Computation, vol. 210, no. 2, pp. 564-570, 2009.

[28] Ju H. Park, O. M. Kwon, and S. M. Lee, "State estimation for neural networks of neutral-type with interval time-varying delays," Applied Mathematics and Computation, vol. 203, no. 1, pp. 217-223, 2008.

[29] K. Hirasawa, S. Mabu, and J. Hu, "Propagation and control of stochastic signals through universal learning networks," Neural Networks, vol. 19, no. 4, pp. 487-499, 2006.

[30] U.S.-Canada Power System Outage Task Force, "Final Report on the August 14th Blackout in the United States and Canada: Causes and Recommendations," April 2004, http:/ /www.nerc.com/.

[31] A. Ray, "Output feedback control under randomly varying distributed delays," Journal of Guidance, Control, and Dynamics, vol. 17, no. 4, pp. 701-711, 1994.

[32] Z. Wang, F. Yang, D. W. C. Ho, and X. Liu, "Robust Hळ filtering for stochastic time-delay systems with missing measurements," IEEE Transactions on Signal Processing, vol. 54, no. 7, pp. 2579-2587, 2006.

[33] Z. Wang, D. W. C. Ho, and X. Liu, "Robust filtering under randomly varying sensor delay with variance constraints," IEEE Transactions on Circuits and Systems II, vol. 51, no. 6, pp. 320-326, 2004. 